\title{
Cervicitis and urethritis caused by group B streptococcus: case report
}

\author{
GEORGE BUTTIGIEG \\ From the Royal Samaritan Hospital for Women, Glasgow
}

SUMMARY Group B streptococcus is not often isolated by gynaecologists when a clinical diagnosis of cervicitis is made. I report on a patient suffering from cervicitis and urethritis caused by this organism, whose symptoms completely resolved after she was treated with phenoxymethylpenicillin.

\section{Introduction}

Group B streptococcus has been implicated in many recorded instances of pelvic inflammatory disease, often puerperal, as well as in urinary tract infections. ${ }^{1}$ It is less well known, although definitely established, as a cause of cervicitis. Only one report of urethritis caused by this organism has, however, been published. ${ }^{2}$ I present a case of cervicitis and urethritis caused by group B streptococcus.

\section{Case report}

A nulliparous girl aged 18 was referred with a history of persistent vaginal discharge that had lasted four months. Intermittent dysuria during the previous month had also been noted. Repeated courses of oral metronidazole and clotrimazole vaginal pessaries had produced no relief whatsoever. The patient had been receiving no other drug treatment but had been taking oral contraceptives for two years. She was unmarried, and had not had sexual intercourse since parting from her boyfriend two weeks previously.

Examination disclosed a foul smelling discharge at the vaginal introitus and an inflamed external urethral meatus. The vagina looked healthy, but a heavy mucopurulent discharge coated the cervix, which looked inflamed and bled easily to touch by an Ayre's spatula. Gram stained smears of material from the urethra and cervix showed pus cells and Gram positive cocci, both being much more preponderant in the cervical smear. No Gram negative diplococci could be seen. Material for culture for

Address for reprints: Dr G Buttigieg, 83 Hyndland Road, Glasgow G12 9JE

Accepted for publication 14 January 1985
Neisseria gonorrhoeae and Chlamydia trachomatis was taken from the urethra and cervix, and a serum sample was examined for chlamydial antibodies. A saline mount of the discharge showed pus cells but no trichomonads. Pelvic examination showed no signs of pelvic inflammatory disease. A midstream specimen of urine was also taken for culture for eubacteria. In view of the failure of treatment with metronidazole and clotrimazole as well as the possible presence of gonococci (in spite of negative microscopy), all medication was withheld until the results of the tests were available.

Both the cervical and urethral cultures grew group B streptococci, which were sensitive to benzyl penicillin and erythromycin. Cultures for $N$ gonorrhoeae and $C$ trachomatis gave negative results, antibodies against chlamydiae were not found, and the midstream specimen of urine gave negative culture results. A seven day course of phenoxymethylpenicillin $250 \mathrm{mg}$ by mouth every six hours was prescribed. When the patient was reviewed two weeks later she had full remission of symptoms and repeat cultures from the previously infected sites gave negative results.

\section{Discussion}

The most interesting aspect of the case presented here was the presence of urethritis caused by group B streptococcus, which (with the cervicitis) remitted completely on treatment with penicillin. In the absence of $N$ gonorrhoeae and in the presence of group B streptococcus, the satisfactory response to penicillin may be taken as evidence that the latter organism was indeed responsible for the urethritis (and the cervicitis). Penicillin would not eradicate a chlamydial infection (which was in any case excluded 
by negative culture and immunofluorescent antibody studies) and would be ineffective against Ureaplasma urealyticum, the other organism relatively commonly implicated in non-gonococcal urethritis. The spread of the infection is most likely to have been by sexual intercourse. $^{3}$

The case presented here is also a salutary reminder that other organisms than the commonly encountered ones should be kept in mind when investigating patients with vaginal discharge presenting to gynaecology departments. ${ }^{4}$
References

1. Pass MA, Gray BM, Dillon HC Jun. Puerperal and perinatal infections with group B streptococci. Am J Obstet Gynecol 1982; 143: 147-52.

2. Chowdhury MNH, Pareek SS. Urethritis caused by group B streptococci: a case report. British Journal of Venereal Diseases 1984;60:56-7.

3. Franciosi RA, Knostman JD, Zimmerman RA. Group B streptococcal neonatal and infant infections. $J$ Pediatr 1973;82:707-18.

4. Pheifer TA, Forsyth PS, Durfee MA, Pollock HM, Holmes, KK. Nonspecific vaginitis: role of Haemophilus vaginalis and treatment with metronidazole. $N$ Engl J Med 1978;298: 1429-34. 